\title{
MODIFIED HHT METHOD USAGE FOR VEHICLE VIBRATION ANALYSIS IN TIME DOMAIN
}

The paper deals with the analysis of vehicle vibrations in the time domain. The main aim is to modify the HHT-method for the solution of mechanical systems with nonlinear members. The modification enables the computation with the constant system computational matrix. This causes that the triangulation is to be performed once only and the nonlinear members affect only the right side of the algebraic equations system. This can dramatically decrease the time consumption of the computation. We used the method modified in this way for the dynamic analysis of the model with the parameters of the specimen "ERRI - vehicle" that was kinematically excited.

Key words: vibration analysis, time domain, modified HHT-method

\section{Introduction}

The analysis of mechanical systems (for example mechanical systems of vehicles) vibration is permanently very topical. The vehicle dynamic properties are determined with the help of this analysis during a new vehicle design, or a renewal of an older existing vehicle. The Eigen frequencies are characteristic of a vehicle construction. A vehicle mechanical system is excited with various types of loads in the operation and this is the reason why its individual parts oscillate. The aim of a dynamical analysis is not only to judge the influence of an excitation on the mechanical system but also, on the base of that analysis, to propose and to perform construction changes of a vehicle for the detected negative state elimination or improvement.

\section{Vehicle vibration analysis}

The aim of the vehicle vibration analysis may be the vehicle behaviour prediction before a performance of experimental tests for a vehicle approving into the operation and saving the time and financial expenses that may arise according to the tests repetition, in the case when previous tests had failed. The vibration analysis with the help of computations allows simulation of extreme loadings that evoke critical states without material losses, negative influence of a vehicle on the track, environment and so on. These computations are in practice often executed with commercial accessible programme packages. They provide results of analysis on the base of chosen vehicle parameters, track parameters and loads. The programmes utilise various methods which are their internal components. It has not become usual that a common user can affect them.

\section{Methods for vibration analysis in the time domain}

The direct numerical integration of the equation of motion is widely used for the linear systems solutions where the individual process character monitoring is important. The principal idea of a numerical integration lies in the fact that we will fulfil the equation of motion in the finite $t_{0}, t_{1}, \ldots, t_{m}$ moments. The distance of individual moments is $\Delta t_{k}=t_{k+1}-t_{k}$, where $k=0,1, \ldots, m-1$, is labeled a length of integration step. Starting conditions are an inseparable part of an equation of motion. We consider the start to be the time $t=0$. Then it holds:

$$
q(0)=q_{0} \quad \dot{q}(0)=\dot{q}_{0}
$$

We distinguish the three types of direct integration numerical methods:

- explicit,

- implicit,

- predictor - corrector.

We consider the first two types to be the fundamental ones. The Predictor - corrector method is in fact a simulation of the implicit method and is suitable for nonlinear systems solutions. The Integration method may be explicit or implicit in dependence on the moment in which it uses the equation of movement.

We compute the $q_{t+\Delta t}, \dot{q}_{t+\Delta t}, \ddot{q}_{t+\Delta t}$ vectors from the equation of motion with the help of an explicit method on the base of the $q, \dot{q}, \ddot{q}$ moving characteristics course shape presupposition in the interval $\langle t, t+\Delta t\rangle$ and their knowledge in the time moment $t$.

\footnotetext{
* Juraj Gerlici, Tomas Lack

Faculty of Mechanical Engineering, University of Zilina, Slovakia, E-mail: juraj.gerlici@fstroj.uniza.sk.
} 
The implicit methods, vice versa, use the equation of motion in the time $t+\Delta t$. They are suitable, first of all, for the solution of the linear equation of motion with a consistent mass matrix.

The stability is a characteristic feature of numerical methods for a direct integration of an equation of motion. The stability means that the solutions must not go through limits for the arbitrary starting conditions. If this condition of an arbitrary ratio of $\Delta t / T_{n}$ is fulfiled, we describe the method as unconditionally stable and if it is fulfiled for an individual critical ratio $\Delta t / T_{n}$ only, we talk about an conditionally stable method. In the following text we will apply some methods that may be used for the mechanical system vibration analysis in the time domain. They are: the Difference method, the Linear acceleration method, the Wilson $\theta$ - method, the Newmark method [3], and the HHT-method [3, 6]

\subsection{The differential method}

For the numerical integration of the equation of motion we substitute the time invariant variable for differenties. The simpliest differential substitutions of the accelerations vector $\ddot{q}$ and the velocities vector $\dot{q}$ in the time t are:

$$
\begin{aligned}
& \dot{q}=\frac{1}{2 \cdot \Delta t} \cdot\left(q_{t+\Delta t}-q_{t-\Delta t}\right), \\
& \ddot{q}=\frac{1}{\Delta t^{2}} \cdot\left(q_{t+\Delta t}-2 \cdot q_{t}+q_{t-\Delta t}\right),
\end{aligned}
$$

that we apply to the equation of motion in the time $t$

$$
M \cdot \ddot{q}_{t}+B \cdot \dot{q}_{t}+K \cdot q_{t}=F_{t} .
$$

After insertion and modification we obtain:

$$
\begin{aligned}
& \left(\frac{1}{\Delta t^{2}} \cdot M+\frac{1}{2 \cdot \Delta t} \cdot B\right) \cdot q_{t+\Delta t}=F_{t}-\left(K-\frac{2}{\Delta t^{2}} \cdot M\right) \cdot q_{t}- \\
& -\left(\frac{1}{\Delta t^{2}} \cdot M-\frac{1}{2 \cdot \Delta t} \cdot B\right) \cdot q_{t+\Delta t} .
\end{aligned}
$$

The differential method is the type of an explicit method. The method has all the advantages of explicit methods, if the damping matrix is $B=0$ or $B=\alpha \cdot M$. The most effective usage is in the case of a diagonal mass matrix, id est for solutions of systems with concentrated massess. The method is only conditionally stable, in this case the lenght of integration step $\Delta t$ has to meet the following demand:

$$
\Delta t \leq \frac{T_{n}}{\pi}
$$

where $T_{n}$ is the smallest period of the vibration. The relatively very short length of integration step is needed in order to ensure that the difference method gives the correct solution. Another disadvantage is the fact, that we need to use the special procedure in the first step.

$$
q_{-\Delta t}=q_{0}-\Delta t \cdot \dot{q}_{0}+\frac{\Delta t^{2}}{2} \cdot \ddot{q}_{0}
$$

Its advantage is the possibility to use it for a nonlinear tasks solution as well.

\subsection{The method of linear acceleration}

This method is based on the presupposition of a linear acceleration course during each of integration steps, as shown in Fig.1.

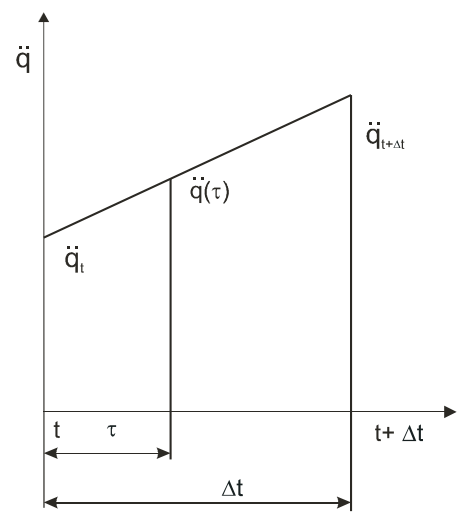

Fig. 1 An acceleration course

We label the variable that changes in the interval $\langle 0, \Delta t\rangle$ with the mark $\tau$, we express the relation for acceleration with:

$$
\ddot{q}(t+\tau)=\ddot{q}_{t}+\frac{\tau}{\Delta t}\left(\ddot{q}_{t+\Delta t}-\ddot{q}_{t}\right)
$$

We obtain the relations for $\dot{q}(t+\tau)$ and $q(t+\tau)$ via the double integration:

$$
\begin{aligned}
& \dot{q}(t+\tau)=\tau \cdot \ddot{q}_{t}+\frac{\tau^{2}}{2 \cdot \Delta t}\left(\ddot{q}_{t+\Delta t}-\ddot{q}_{t}\right)+\dot{q}_{t} \\
& q(t+\tau)=\frac{\tau^{2}}{2} \cdot \ddot{q}_{t}+\frac{\tau^{3}}{6 \cdot \Delta t}\left(\ddot{q}_{t+\Delta t}-\ddot{q}_{t}\right)+\tau \cdot \dot{q}_{t}+q_{t}
\end{aligned}
$$

In the end the integration step for $\tau=\Delta t$ we will get:

$$
\begin{aligned}
& \dot{q}_{t+\Delta t}=\Delta t \cdot \ddot{q}_{t}+\frac{\Delta t}{2}\left(\ddot{q}_{t+\Delta t}-\ddot{q}_{t}\right)+\dot{q}_{t}=\dot{q}_{t}+\frac{\Delta t}{2}\left(\ddot{q}_{t+\Delta t}+\ddot{q}_{t}\right) \\
& q_{t+\Delta t}=\frac{\Delta t^{2}}{2} \ddot{q}_{t}+\frac{\Delta t^{2}}{6}\left(\ddot{q}_{t+\Delta t}-\ddot{q}_{t}\right)+\Delta t \cdot \dot{q}_{t}+q_{t}= \\
& =q_{t}+\Delta t \cdot \dot{q}_{t}+\frac{\Delta t^{2}}{6}\left(\ddot{q}_{t+\Delta t}+2 \cdot \ddot{q}_{t}\right)
\end{aligned}
$$

From these relations we obtain the relations for $\ddot{q}_{t+\Delta t}$ a $\dot{q}_{t+\Delta t}$

$$
\begin{aligned}
& \ddot{q}_{t+\Delta t}=\frac{6}{\Delta t^{2}}\left(q_{t+\Delta t}-\Delta t \cdot \dot{q}_{t}-q_{t}\right)-2 \cdot \ddot{q}_{t} \\
& \dot{q}_{t+\Delta t}=\dot{q}_{t}+\frac{3}{\Delta t}\left(q_{t+\Delta t}-\Delta t \cdot \dot{q}_{t}-q_{t}\right)-\Delta t \cdot \ddot{q}_{t}+\frac{\Delta t}{2} \ddot{q}_{t}= \\
& =\frac{3}{\Delta t} \cdot q_{t+\Delta t}-2 \cdot \dot{q}_{t}-\frac{3}{\Delta t} \cdot q_{t}-\frac{\Delta t}{2} \ddot{q}_{t}
\end{aligned}
$$


After the insertion of the previous relations into the equation of motion in the time $t+\Delta t$ and after modification the equation obtains the form:

$$
\begin{aligned}
& \left(\frac{6}{\Delta t^{2}} M+\frac{3}{\Delta t} B+K\right) \cdot q_{t+\Delta t}= \\
& =F_{t+\Delta t}+M\left(\frac{6}{\Delta t^{2}} q_{t}+\frac{6}{\Delta t^{2}} \dot{q}_{t}+2 \ddot{q}_{t}\right)+ \\
& +B\left(\frac{3}{\Delta t} q_{t}+2 \dot{q}_{t}+\frac{\Delta t}{2} \ddot{q}_{t}\right)
\end{aligned}
$$

The method of the linear acceleration does not request any starting procedure because displacements, velocities and accelerations in the time $t+\Delta t$ are expressed in the dependence on the same quantities in the time $t$.

\subsection{The Wilson $\theta$-method}

Similar to the Newmark method, the Wilson method is implicit as well. Its original version, the method of linear acceleration, is in fact the Newmark method, where $\delta=1 / 2$ and $\alpha=1 / 6$. The method of linear acceleration is a special case of the Wilson $\theta$-method, where $\theta=2$. The linear change of acceleration in the interval $\langle t, t+\theta \Delta t\rangle$ is the basic presupposition, as is shown in Fig. 2. In the arbitrary point of the interval the following holds:

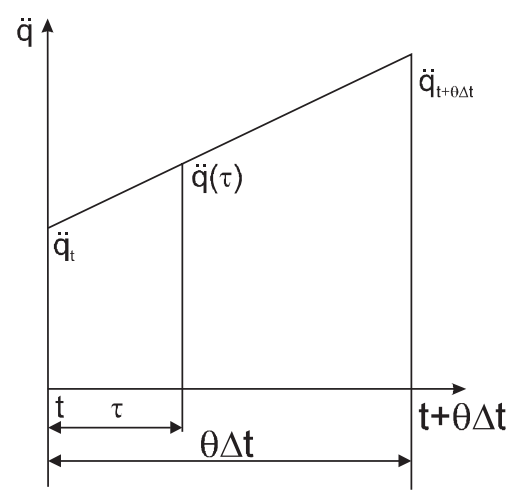

Fig. 2 An acceleration dependence on the time

$$
\ddot{q}(t+\tau)=\ddot{q}_{t}+\frac{\tau}{\theta \cdot \Delta t}\left(\ddot{q}_{t+\theta \Delta t}-\ddot{q}_{t}\right)
$$

Via a double integration we obtain the relations for $\dot{q}(t+\tau)$ and $q(t+\tau)$ :

$$
\begin{aligned}
& \dot{q}(t+\tau)=\dot{q}_{t}+\tau \ddot{q}_{t}+\frac{\tau^{2}}{2 \cdot \theta \cdot \Delta t}\left(\ddot{q}_{t+\theta \Delta t}-\ddot{q}_{t}\right) \\
& q(t+\tau)=q_{t}+\tau \dot{q}_{t}+\frac{\tau^{2}}{2} \ddot{q}_{t}+\frac{\tau^{3}}{6 \cdot \theta \cdot \Delta t}\left(\ddot{q}_{t+\theta \Delta t}-\ddot{q}_{t}\right)
\end{aligned}
$$

At the end of an integration step for $\tau=\theta \cdot \Delta t$ we have:

$$
\begin{aligned}
& \dot{q}_{t+\theta \Delta t}=q_{t}+\theta \cdot \Delta t \cdot \ddot{q}_{t}+\frac{\theta \cdot \Delta t}{2}\left(\ddot{q}_{t+\theta \Delta t}-\ddot{q}_{t}\right)= \\
& =\dot{q}_{t}+\frac{\theta \cdot \Delta t}{2}\left(\ddot{q}_{t+\theta \Delta t}-\ddot{q}_{t}\right)
\end{aligned}
$$

$$
\begin{aligned}
& q_{t+\theta \Delta t}=q_{t}+\theta \cdot \Delta t \cdot \dot{q}_{t}+\frac{\theta^{2} \cdot \Delta t^{2}}{2} \ddot{q}_{t}+\frac{\theta^{2} \cdot \Delta t^{2}}{6}\left(\ddot{q}_{t+\theta \Delta t}-\ddot{q}_{t}\right)= \\
& =q_{t}+\theta \cdot \Delta t \cdot \dot{q}_{t}+\frac{\theta^{2} \cdot \Delta t^{2}}{6}\left(\ddot{q}_{t+\theta \Delta t}+2 \cdot \ddot{q}_{t}\right)
\end{aligned}
$$

These relations lead to the expressions for $\ddot{q}_{t+\theta \Delta t}$ a $\dot{q}_{t+\theta \Delta t}$

$$
\begin{aligned}
& \ddot{q}_{t+\theta \Delta t}=\frac{6}{\theta^{2} \cdot \Delta t^{2}}\left(q_{t+\theta \Delta t}-\theta \cdot \Delta t \cdot \dot{q}_{t}-q_{t}\right)-2 \cdot \ddot{q}_{t} \\
& \dot{q}_{t+\theta \Delta t}=\dot{q}_{t}+\frac{3}{\theta \cdot \Delta t}\left(q_{t+\theta \Delta t}-\theta \cdot \Delta t \cdot \dot{q}_{t}-q_{t}\right)- \\
& -\theta \cdot \Delta t \cdot \ddot{q}_{t}+\frac{\theta \cdot \Delta t}{2} \cdot \ddot{q}_{t}=\frac{3}{\theta \cdot \Delta t} q_{t+\theta \Delta t}-2 \cdot \dot{q}_{t}- \\
& -\frac{3}{\theta \cdot \Delta t} q_{t}-\frac{\theta \cdot \Delta t}{2} \cdot \ddot{q}_{t}
\end{aligned}
$$

We insert the previous relations into the equation of motion in the time $\tau=\theta \cdot \Delta t$ and after the modification the equation gets the form:

$$
\begin{aligned}
& \left(\frac{6}{\theta^{2} \cdot \Delta t^{2}} M+\frac{3}{\theta \cdot \Delta t} B+K\right) \cdot q_{t+\theta \Delta t}=F_{t+\theta \Delta t}+ \\
& +M\left(\frac{6}{\theta^{2} \cdot \Delta t^{2}} \cdot q_{t}+\frac{6}{\theta \cdot \Delta t} \cdot \dot{q}_{t}+2 \cdot \ddot{q}_{t}\right)+ \\
& +B\left(\frac{3}{\theta \cdot \Delta t} \cdot q_{t}+2 \cdot \dot{q}_{t}+\frac{\theta \cdot \Delta t}{2} \cdot \ddot{q}_{t}\right)
\end{aligned}
$$

The stability and the accuracy of the method depends on the coefficient $\theta$ choice. For the method stability it is necessary to be $\theta \geq 1.37$.

\subsection{The Newmark method}

This method is an implicit method. It comes out from the method of the average constant acceleration. The principal retations of the Newmark method give the relationship between the displacement, the velocity and the acceleration in the $t$ and $t+\Delta t$. They are:

$$
\begin{aligned}
& \dot{q}_{t+\Delta t}=\dot{q}_{t}+\Delta t \cdot\left[\delta \cdot \ddot{q}_{t+\Delta t}+(1-\delta) \ddot{q}_{t}\right] \\
& q_{t+\Delta t}=q_{t}+\Delta t \cdot \dot{q}_{t}+\left[\alpha \cdot \ddot{q}_{t+\Delta t}+\left(\frac{1}{2}-\alpha\right) \ddot{q}_{t}\right] \cdot \Delta t^{2}
\end{aligned}
$$

From the previous relations we obtain the expressions for $\ddot{q}_{t+\Delta t}$ and $\dot{q}_{t+\Delta t}$

$$
\begin{aligned}
& \ddot{q}_{t+\Delta t}=\frac{1}{\alpha \cdot \Delta t^{2}}\left(q_{t+\Delta t}-\Delta t \cdot \dot{q}_{t}-q_{t}\right)-\left(\frac{1}{2 \cdot \alpha}-1\right) \ddot{q}_{t} \\
& \dot{q}_{t+\Delta t}=\frac{\delta}{\alpha \cdot \Delta t} q_{t+\Delta t}-\left(\frac{\delta}{\beta}-1\right) \dot{q}_{t}-\frac{\delta}{\alpha \cdot \Delta t} q_{t}- \\
& -\Delta t\left(\frac{\delta}{2 \cdot \alpha}-1\right) \ddot{q}_{t}
\end{aligned}
$$

Inserting the previous relations into the equation of motion in the time $t+\Delta t$ and after the modification, the equation has the form: 


$$
\begin{aligned}
& \left(\frac{1}{\alpha \cdot \Delta t^{2}} \cdot M+\frac{\delta}{\alpha \cdot \Delta t} \cdot B+K\right) q_{t+\Delta t}=F_{t+\Delta t}+ \\
& +B\left[\frac{\delta}{\alpha \cdot \Delta t} q_{t}+\left(\frac{\delta}{\alpha}-1\right) \dot{q}_{t}+\Delta t \cdot\left(\frac{\delta}{2 \cdot \alpha}-1\right) \ddot{q}_{t}\right]+ \\
& +M\left[\frac{1}{\alpha \cdot \Delta t^{2}} q_{t}+\frac{1}{\alpha \cdot \Delta t} \dot{q}_{t}+\left(\frac{1}{2 \cdot \alpha}-1\right) \ddot{q}_{t}\right]
\end{aligned}
$$

where:

$$
\delta=\frac{1}{2}, \quad \alpha=\frac{1}{4} .
$$

\subsection{HHT method}

The HHT method [6] comes directly out from the Newmark method, where we create for $\ddot{q}_{n+1}$ and $\dot{q}_{n+1}$ the relations of:

$$
\begin{aligned}
& \ddot{q}_{n+1}=a_{0}\left(q_{n+1}-q_{n}\right)-a_{2} \cdot \dot{q}_{n}-a_{3} \cdot q_{n} \\
& \dot{q}_{n+1}=a_{1}\left(q_{n+1}-q_{n}\right)-a_{4} \cdot \dot{q}_{n}-a_{5} \cdot \ddot{q}_{n} .
\end{aligned}
$$

The principal equation is

$$
\begin{aligned}
& M \ddot{q}_{n+1}+\left(1-\alpha_{f}\right) C \dot{q}_{n+1}+\alpha_{f} C \dot{q}_{n}+ \\
& +\left(1-\alpha_{f}\right) K q_{n+1}+\alpha_{f} K q_{n}=F\left(\tilde{t}_{n+1}\right)
\end{aligned}
$$

where

$$
\tilde{t}_{n+1}=t_{n}+\left(1-\alpha_{f}\right) \Delta t
$$

We put the relations (24) and (25) into the (26) and we get:

$$
\begin{aligned}
& {\left[a_{0} \cdot M+a_{1} \cdot B+\left(1-\alpha_{f}\right) \cdot K\right] \cdot q_{n+1}=F_{n+1}^{A}-} \\
& -\alpha_{f} \cdot K \cdot q_{n}+M \cdot\left[a_{0} \cdot q_{n}+a_{2} \cdot \dot{q}_{n}+a_{3} \cdot \ddot{q}_{n}\right]+ \\
& +B \cdot\left[a_{1} \cdot q_{n}+a_{4} \cdot \dot{q}_{n}+a_{5} \cdot \ddot{q}_{n}\right]
\end{aligned}
$$

where

$F_{n+1}^{A}=\left(1-\alpha_{f}\right) \cdot F_{n+1}+\alpha_{f} \cdot F_{n}$ is an applied load

$a_{0}=\frac{1-\alpha_{m}}{\alpha \cdot \Delta t^{2}} \quad a_{2}=\frac{1-\alpha_{m}}{\alpha \cdot \Delta t} \quad a_{4}=\frac{\left(1-\alpha_{f}\right) \cdot \delta}{\alpha}-1$

$a_{1}=\frac{\left(1-\alpha_{f}\right) \cdot \delta}{\alpha \cdot \Delta t} \quad a_{3}=\frac{1-\alpha_{m}}{2 \cdot \alpha}-1$

$a_{5}=\left(1-\alpha_{f}\right) \cdot\left(\frac{\delta}{2 \cdot \alpha}-1\right) \cdot \Delta t$

The basic setting is

$\alpha=\frac{1}{4} \cdot(1+\gamma)^{2} \quad \delta=\frac{1}{2}+\gamma \quad \alpha_{f}=\gamma \quad \alpha_{m}=0$

$\gamma$ - is the coefficient of an amplitude damping; it may reach the value $\gamma=0 \rightarrow \frac{1}{3}$.
It is possible to change the setting according to the conditions.

$$
\delta \geq \frac{1}{2} \quad \alpha \geq \frac{1}{2} \cdot \delta \quad \delta=\frac{1}{2}-\alpha_{m}+\alpha_{f} \quad \alpha_{m} \leq \alpha_{f} \leq \frac{1}{2}
$$

If we use these alternatives, we determine two other methods for the parameters determination. We can choose the given coefficient of an amplitude damping $\gamma$ for four integration parameters in the following way [8].

$$
\alpha=\frac{1}{4} \cdot(1+\gamma)^{2} \delta=\frac{1}{2}+\gamma \quad \alpha_{f}=0 \quad \alpha_{m}=-\gamma,
$$

or we can use the procedure via Chung and Hulbert [3]:

$$
\alpha_{f}=\frac{1-\gamma}{2} \quad \alpha_{m}=\frac{1-3 \cdot \gamma}{2} .
$$

\section{Modified HHT for the solution of systems with nonlinear members [4]}

$$
\begin{aligned}
& {\left[a_{0} \cdot M+a_{1} \cdot B+\left(1-\alpha_{f}\right) \cdot K\right] \cdot q_{n+1}=F_{n+1}^{A}-\alpha_{f} \cdot K \cdot} \\
& \cdot q_{n}+F_{n+1}^{K+}+F_{n+1}^{B+}+M\left[a_{0} \cdot q_{n}+a_{2} \cdot \dot{q}_{n}+a_{3} \cdot \ddot{q}_{n}\right]+ \\
& +B\left[a_{1} \cdot q_{n}+a_{4} \cdot \dot{q}_{n}+a_{5} \cdot \ddot{q}_{n}\right]
\end{aligned}
$$

where

$F_{n+1}^{A}$ is an applied loading

$$
F_{n+1}^{A}=\left(1-\alpha_{f}\right) \cdot F_{n+1}+\alpha_{f} \cdot F_{n}
$$

$F_{n+1}^{K+}$ is the additional force of nonlinear springs

$$
F_{n+1}^{K+}=F_{n}^{K+}\left(q_{n}\right) \cdot\left(1+\alpha_{f}\right)-F_{n-1}^{K+}\left(q_{n-1}\right) \cdot \alpha_{f}
$$

$F_{n+1}^{B+}$ is the additional force of the nonlinear dampers

$$
F_{n+1}^{B+}=F_{n}^{B+}\left(\dot{q}_{n}\right) \cdot\left(1-\alpha_{f}\right)+F_{n-1}^{B+}\left(\dot{q}_{n-1}\right) \cdot \alpha_{f}
$$

All the computations in the time domain were performed by the HHT method which was modified for nonlinear members with the following parameters $\gamma=0.15, \alpha_{m}=0.15$ and $\Delta_{t}=0.005 \mathrm{sec}$. computation.

The $F_{k}^{K+}$ and $F_{k}^{B+}$ for a nonlinear spring and a nonlinear damper determination respectively

For a nonlinear spring the following holds:

$$
\begin{aligned}
& K_{k}=T^{T} \cdot\left[\begin{array}{cc}
k & -k \\
-k & k
\end{array}\right] \cdot T \\
& F_{k}^{K+}=T^{T} \cdot\left[\begin{array}{c}
f(d)-k \cdot d \\
-(f(d)-k \cdot d)
\end{array}\right] \cdot T
\end{aligned}
$$


where:

$k \quad-$ is the basic spring stiffness,

$T$ - is the transformation matrix for the transformation from a local into a global coordinate system,

$K_{k}$ - is the element stiffness matrix,

$d$ - is the strain of a spring in the local coordinate system of the spring,

$f(d)$ - is the prescribed functional dependence of an internal force and strain in a spring,

$F_{k}^{K+}-$ is the additional force from a nonlinear spring.

For a nonlinear damper the following holds:

$$
\begin{aligned}
& B_{k}=T^{T} \cdot\left[\begin{array}{cc}
b & -b \\
-b & b
\end{array}\right] \cdot T \\
& F_{k}^{B+}=T^{T} \cdot\left[\begin{array}{c}
f(v)-b \cdot v \\
-(f(v)-b \cdot v)
\end{array}\right] \cdot T
\end{aligned}
$$

where:

$b$ - is the basic damping constant of a damper,

$T$ - is the transformation matrix for a transformation from a local into a global coordinate system,

$B_{k}$ - is the matrix of an element damping,

$v \quad$ - is the mutual velocity of node points of a damper in the local coordinate system of a damper,

$f(v)$ - is the prescribed functional dependence of an internal force in a damper and a mutual velocity of node points of a damper in the local coordinate system of a damper,

$F_{k}^{B+}$ - is the additional force from a nonlinear damper.

\section{Test of modified HHT-method}

For this method validation we used the comparison of computer evaluated dependence courses of forces and velocities, or

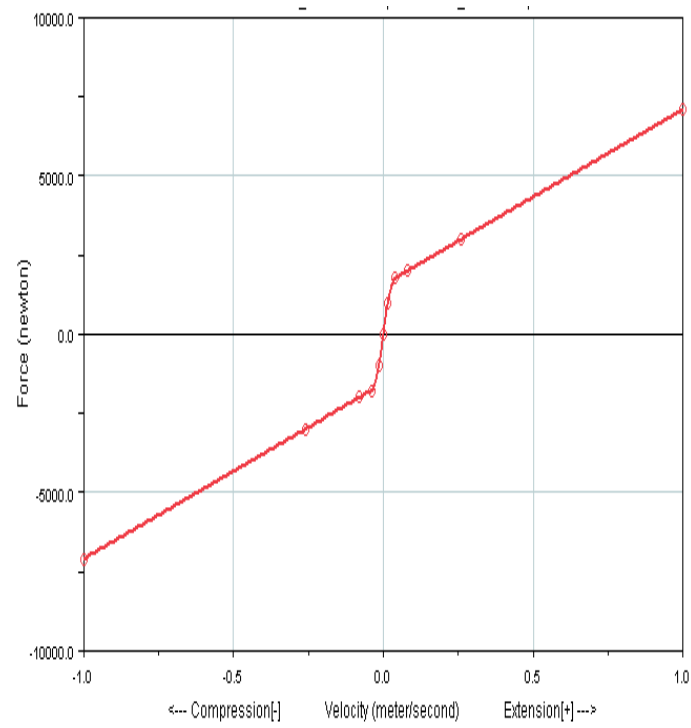

deformation "measured" in nonlinear members of computational model flexible bindings and given teoretical characteristics. These nonlinear courses may in fact describe the properties of dampers and springs. The example of a bogie model that would be equiped with flexible bindings and dampers with nonlinear characteristics is depicted in Fig. 3.

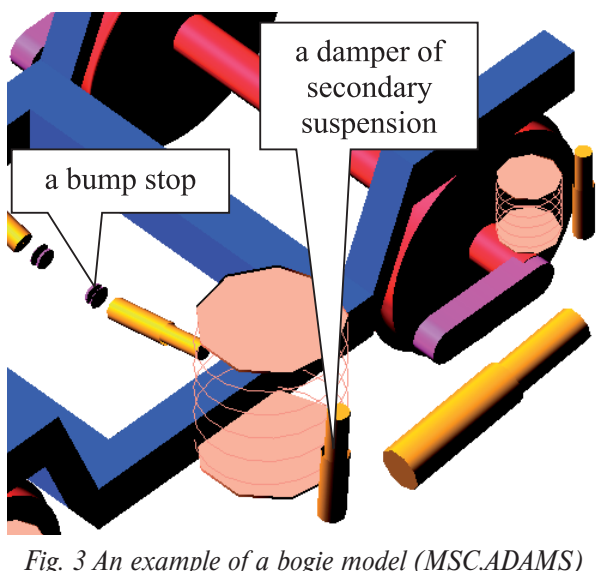

The principle: the forces in nonlinear members were computed as the internal forces in the serial connected linear springs with high stiffness. The prescribed flexible member characteristics are represented by curves in the graphs. The linear dependency represents the basic stiffness, or better, the basic stiffness constant.

\section{Conclusion}

Simulation computations mainly supported by computational technique are used for the vehicle vibration analysis. The procedures and methods are used for the forced oscillation of a mechanical

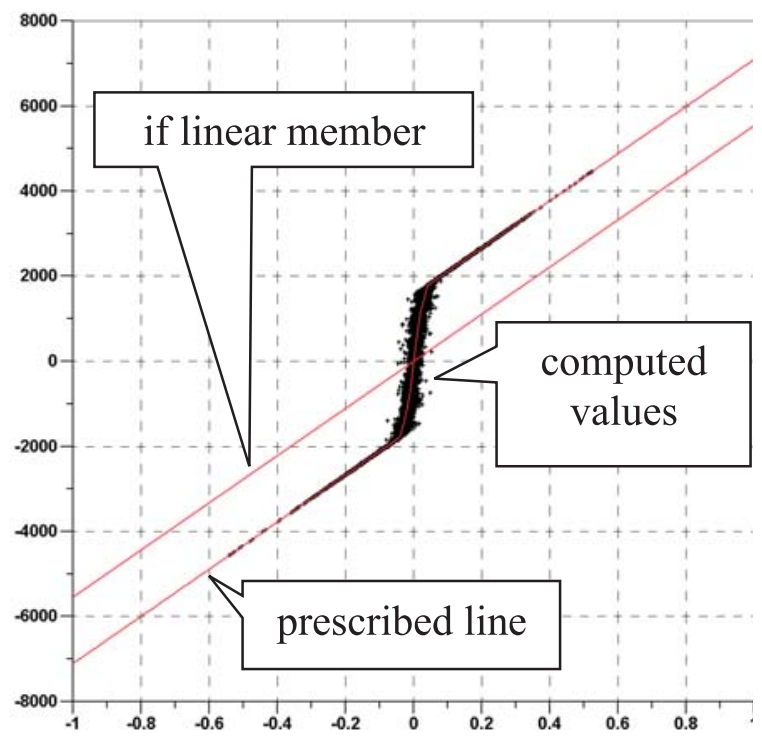

Fig. 4 A secondary suspension damper (left: given parameters, right: a comparison) 


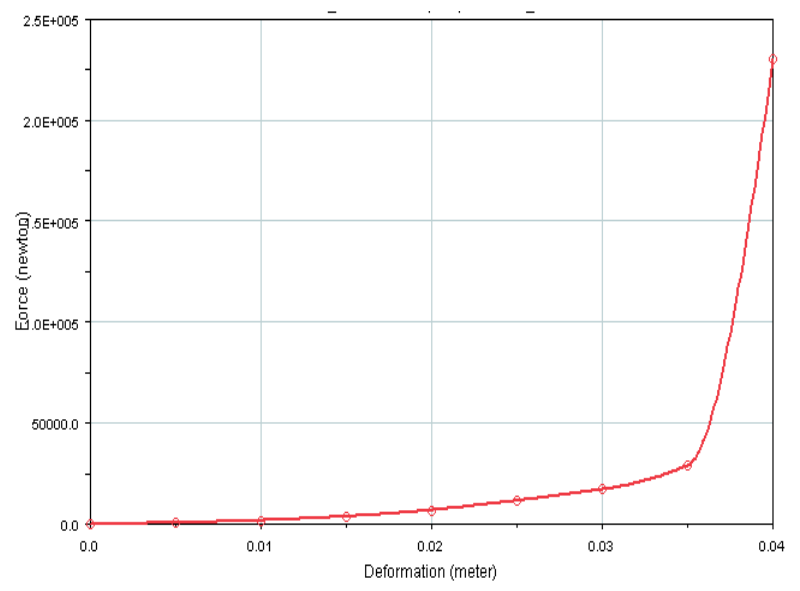

Fig. 5 A prescribed characteristics

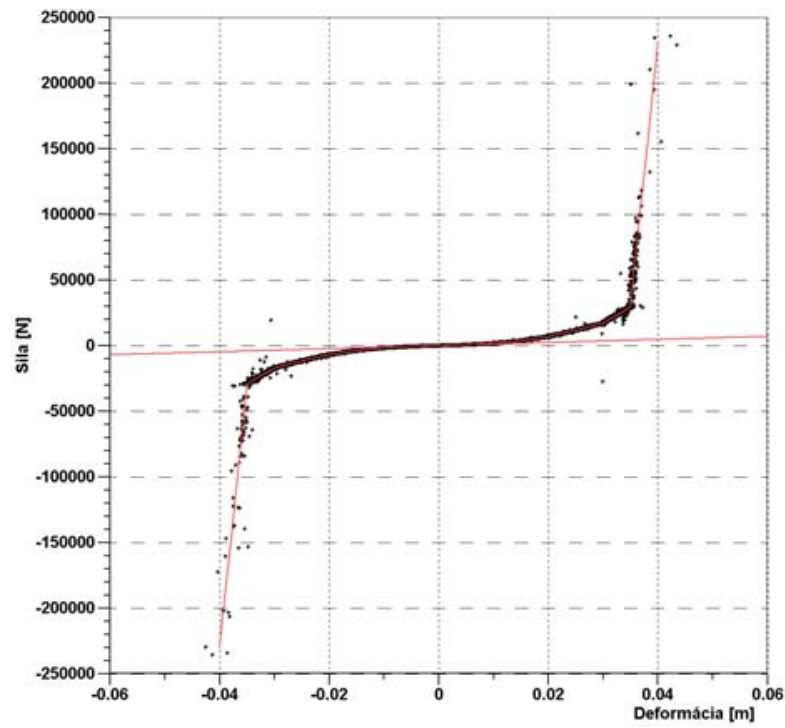

Fig. 6 An internal force versus a deformation

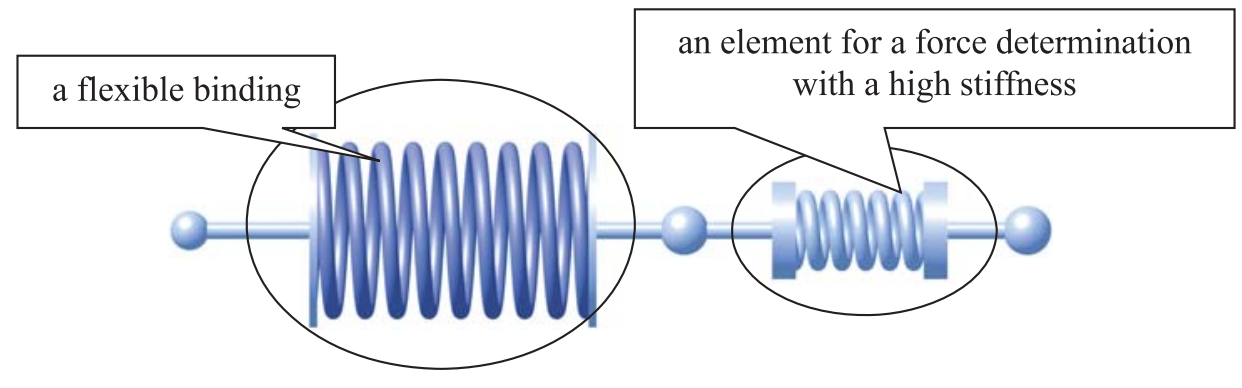

Fig. 7 Schematic depiction of "measurement" principle of internal forces in a flexible binding

system solution. The vehicle model was created for this purpose [4]. A kinematic excitation and accelerations act on the model. We can search a response from a harmonic excitation via the solution in a complex or real form, or we can analyse the vehicle vibration in the time domain. It is useful to use the HHT method [7] as a special variant of the Newmark method. The main aim was to modify the HHT-method for the solution of mechanical systems with nonlinear members. The modification enables the computation with the constant system computational matrix. This causes that the triangulation is to be performed once only and the nonlinear members affect only the right side of the algebraic equations system. This can dramatically decrease time consumption of the computation. We used the method modified in this way for the dynamical analysis of the model with the parameters of the spec- imen "ERRI - vehicle" that was kinematically excited. The procedures were performed with the DELTA programme [5].

\section{Acknowledgement}

The work was supported by the Scientific Grant Agency of the Ministry of Education of the Slovak Republic and the Slovak Academy of Sciences in the project No. 1/3169/06 "Properties Research of Rail Vehicles in Movement with Emphasis on the Solution of a Wheel and Rail Contact at the Wheelset Rolling in the Rail via Computer Simulation" and in the project No. 1/4119/07: "Investigation of a dynamic properties of a vehicle".

\section{References}

[1] GERLICI, J., LACK, T.: An Analysis of Bumpers and Springs Parameters Influence on the Ride Comfort (in Slovak), Dynamics of Rigid and Deformable Bodies, Proc. of V. international conference, ISBN 978-80-7044-914-1, pp. 39-48, Univerzita J. E. Purkyne v Usti nad Labem, 2007. 


\section{COMMNICOIIONS}

[2] GERLICI, J., LACK, T.: Methods for Vehicle Vibration Analysis in Time Domain, Prace naukowe Politechniki Warszawskiej, Z. 63 , Transport, 2007, pp. 71-81, Publishing House of the Warsaw University of Technology, ISSN 1230-9265, Warszawa, 2007.

[3] CHUNG, J., HULBERT, G. M.: A Time Integration Algorithm for Structural Dynamics with Improved Numerical Dissipation: The Generalized-a Method, Journal of Applied Mechanics, ISSN: 0021-8936, Vol. 60, p. 371 (1993).

[4] LACK, T.: The Dynamic Properties Analysis of Vehicles from the Point of View of Ride Comfort (in Slovak), Habilitation Work, pp. 176, Faculty of Mechanical Engineering University of Zilina, 2007.

[5] LACK, T., GERLICI, J.: The Programme System DELTA Exploitation for Numerical Analysis Performances (in Slovak), Proc. of $18^{\text {th }}$ International Conference "Current Problems in Rail Vehicles - PRORAIL 2007" - Part II, pp. 11-22, EDIS, ISBN 978-80-8927607-3, Zilina, 2007.

[6] LACK, T., GERLICI, J.: Vehicles Dynamic Properties Analysis from the Point of View of Comfort for Passengers, Archives of Transport, issue 1-2/2007, pp. 91-110, ISSN 8066-9546, Warszawa, 2007.

[7] NEGRUT, D., RAMPALLI, R., SAJDAK, T.: On the Implementation of the method in MSC.ADAMS, p. 12, 2004, Technical support for MSC.SOFTWARE users, published on the Internet.

[8] WOOD, W. L., BOSSAK, M., ZIENKIEWICZ, O. C.: An Alpha Modification of Newmark Method, International Journal of Numerical Method in Engineering, ISSN 0020-7683, vol. 15, p. 1562, 1981 